Claremont Colleges

Scholarship@ Claremont

All HMC Faculty Publications and Research

HMC Faculty Scholarship

$1-1-1992$

\title{
A CASSCF Study of Various Rotamers of the Hexatriene Radical Cation
}

Robert J. Cave

Harvey Mudd College

Jenifer L. Johnson

Harvey Mudd College

\section{Recommended Citation}

Cave, R.J.; Johnson, J. “A CASSCF study of various rotamers of the hexatriene radical cation,” J. Phys. Chem. 1992, 96, 5332-5336. doi: $10.1021 /$ j100192a029

This Article is brought to you for free and open access by the HMC Faculty Scholarship at Scholarship @ Claremont. It has been accepted for inclusion in All HMC Faculty Publications and Research by an authorized administrator of Scholarship @ Claremont. For more information, please contact scholarship@cuc.claremont.edu. 


\title{
A CASSCF Study of Various Rotamers of the Hexatriene Radical Cation
}

\author{
Robert J. Cave* and Jenifer L. Johnson \\ Department of Chemistry, Harvey Mudd College, Claremont, California 91711 (Received: February 1, 1992)
}

\begin{abstract}
Results are presented from $a b$ initio calculations on the ground and two low-lying excited states of the hexatriene radical cation in a variety of stable conformations of the electronic ground state of the molecule. We have performed Hartree-Fock geometry optimizations using the STO-3G and 6-31G basis sets and have performed vibrational analyses for all stable conformers in the 6-31G basis. In addition, we have performed geometry optimizations in both basis sets using CASSCF wave functions where the five $\pi$ electrons are correlated. No new geometrical information is obtained at this level of treatment. Excitation energies to the two lowest-lying doublet $\pi$ excited states for the six stable ground-state geometries were also calculated using CASSCF wave functions. These excitation energies are compared with previous experimental assignments of observed transitions in photolytically generated hexatriene radical cations.
\end{abstract}

\section{Introduction}

Polyene spectroscopy is an active area of collaboration for experiment and electronic structure theory. In addition to the examination of polyene spectroscopy in the context of studies of visual chromophores' and conducting polymers, ${ }^{2}$ much recent work has been aimed at understanding the relative energy ordering of the low-lying states in short-chain polyenes such as butadiene, hexatriene, and octatraene. ${ }^{3}$ Recent experiments have clearly established that the lowest excited state in cis-hexatriene is the doubly excited state, ${ }^{3 f, g}$ a result that is counterintuitive until one considers the mixing of the various nominally degenerate zeroorder doubly excited configurations. Other recent work on cyclopentadiene also suggests that the lowest excited state is the doubly excited state ${ }^{31}$ with a smaller gap between the first and second excited states than that observed in hexatriene.

While semiempirical theory successfully predicted this ordering 15 years ago, ${ }^{4}$ ab initio electronic structure theory has yet to unequivocally properly order the two lowest excited states of the polyenes. ${ }^{5}$ (However, recent work by Graham and Freed provides quite good agreement with available experimental work on butadiene. ${ }^{6}$ ) These difficulties arise largely from the need to correlate all valence electrons for both low-lying excited states (i.e., neither state can be treated as a $\pi$-electron-only problem) and the large number of valence electrons in the molecules. Semiempirical methods explicitly treat only the $\pi$ electrons but apparently build $\sigma-\pi$ correlation into the effective Hamiltonian, yielding excellent agreement with experiment.

Polyene radical cations have also been an area of interest for experimentalists ${ }^{7}$ and theorists. ${ }^{8}$ Like the neutral species, the low-lying states arise from $\pi \rightarrow \pi^{*}$ excitations and are mixtures of two or more zero-order states. They are somewhat more difficult to treat experimentally due to the problems in generating them and preventing their subsequent reaction, but theoretically they turn out to be a simpler problem than the neutral molecules. ${ }^{8 \mathrm{~d}}$ In the neutral molecules one encounters difficulties brought on by valence-Rydberg mixing, even for the low-lying valence states, since the ionization potentials (IPs) of the neutral polyenes are so low. However, in the cations the IPs are much higher, and as a consequence the Rydberg states lie much higher than the valence states, and their mixing in the low-lying excited states is negligible. $^{8 d}$

Hexatriene radical cation $\left(\mathrm{C}_{6} \mathrm{H}_{8}{ }^{+}\right)$in particular has been the subject of rather detailed experimental treatment. In 1977 Shida et al. ${ }^{9}$ attempted to systematically assign the various rotational conformers (dubbed "rotamers") of hexatriene+ obtained upon ionization of cyclohexadiene and subsequent photolysis of the products in a Freon matrix. On the basis of their spectra and semiempirical calculations on the various rotamers they assigned four of the possible six rotamers of hexatriene ${ }^{+}$(ccc, ctc, tct, and $\mathrm{ttt}$, where $t$ denotes trans, $\mathrm{c}$ denotes cis, and the three letters denote the conformation about the three central bonds). The other two possibilities, ctt and cct, were suggested to not be formed in the

\footnotetext{
* Author to whom correspondence should be addressed.
}

process. Later work by two other groups reexamined these reactions in argon matrices. ${ }^{10,11}$ The work of Kelsall and Andrews ${ }^{10}$ found similar spectra to those obtained by Shida et al., but they were unable to conclude anything further for the assignments. On the other hand, Bally et al. ${ }^{11}$ concluded they had observed five of the six possible rotamers, and provided assignments for these.

In terms of theoretical treatments of this system, a variety of semiempirical methods have been brought to bear on the ttt rotamer (see ref $7 \mathrm{~b}$ and references cited therein), and CNDO/S and INDO calculations were used by Shida et al. ${ }^{9}$ to interpret their experimental results. However, in the latter case steric interactions appear to be qualitatively inaccurate, and excitation energies were obtained with only qualitative accuracy. To our knowledge there has been no ab initio work on any of the rotamers of hexatriene ${ }^{+}$.

We have recently compared a variety of ab initio methods for the treatment of the ground and excited states of butadiene radical cation. ${ }^{8 d}$ One of our conclusions was that while correlation of all valence electrons tended to lower the computed excitation energies, relative to CASSCF calculations correlating only the $\pi$ electrons, no new qualitative information was gained from all-electron correlation, and reasonable quantitative agreement was obtained in most cases. For the treatment of the relative energetics of several conformers and the excitation energies at each of these stationary points, it was considered important to treat all points uniformly. The CASSCF method provides a means of treating all geometries at the same level, allowing the necessary multiconfigurational description of the various excited states, while avoiding the need for perturbative selection of configurations. In essence, in using this method we assume that the neglected correlation of the $\sigma$ electrons has limited differential effeet on the relative energies of the various rotamers or on the relative excitation energies to a given state for different rotamers. In the case of butadiene good evidence exists to suggest that the relative ground-state energies of various conformers are changed only a small amount by inclusion of correlation. ${ }^{12}$

The present study thus has several aims. First, we seek to examine the stability of various hexatriene ${ }^{+}$rotamers and order them energetically. Second, we seek to estimate the relative excitation energies to the first and second $\pi$ excited states of the hexatriene ${ }^{+}$rotamers. That is, while we will not predict the excitation energies to high accuracy, we suggest that the ordering of the excitation energies for the various rotamers will still be basically correct. We then compare our results with experiment and the various assignments that have been made in the past.

The remainder of the paper is organized as follows. In the following section we discuss the theoretical methods used, followed by presentation of the results of our calculations. In section IV the results are discussed and compared with the past experimental and theoretical results, followed by our principal conclusions.

\section{Theoretical Methods}

All geometry optimizations were performed in $C_{1}$ symmetry. The rotamer ttt yielded a $C_{2 h}$ geometry while the tct and ctc 
TABLE I: Geometries for Various Rotamers of $\mathrm{C}_{6} \mathrm{H}_{8}{ }^{+}$

\begin{tabular}{|c|c|c|c|c|c|}
\hline rotamera & $\mathrm{C}_{1}-\mathrm{C}_{2}{ }^{b}$ & $\mathrm{C}_{2}-\mathrm{C}_{3}{ }^{b}$ & $\mathrm{C}_{3}-\mathrm{C}_{4}$ & $\theta_{\mathrm{C}_{1} \mathrm{C}_{2} \mathrm{C}_{3} \mathrm{C}_{4}}$ & $\overline{\theta_{\mathrm{C}_{2} \mathrm{C}_{3} \mathrm{C}_{4} \mathrm{C}_{5}}}$ \\
\hline $\begin{array}{l}\mathrm{ttt} \\
\mathrm{tct} \\
\mathrm{ctt} \\
\mathrm{ctc}\end{array}$ & $\begin{array}{l}1.349 \\
1.348 \\
1.350,1.347 \\
1.348\end{array}$ & $\begin{array}{l}\text { STO-3G ROI } \\
1.430 \\
1.431 \\
1.429,1.436 \\
1.436\end{array}$ & $\begin{array}{l}\mathrm{HF}^{c} \\
1.412 \\
1.416 \\
1.413 \\
1.412\end{array}$ & $\begin{array}{r}180 \\
180 \\
0 \\
0\end{array}$ & $\begin{array}{r}180 \\
0 \\
180 \\
180\end{array}$ \\
\hline $\begin{array}{l}\text { ttt } \\
\text { tct } \\
\text { ctt } \\
\text { ctc }\end{array}$ & $\begin{array}{l}1.382 \\
1.382 \\
1.383,1.381 \\
1.382\end{array}$ & $\begin{array}{l}\text { STO-3G CAS } \\
1.429 \\
1.430 \\
1.428,1.435 \\
1.435\end{array}$ & $\begin{array}{l}\mathrm{SCF}^{c} \\
1.417 \\
1.421 \\
1.417 \\
1.417\end{array}$ & $\begin{array}{r}180 \\
180 \\
0 \\
0\end{array}$ & $\begin{array}{r}180 \\
0 \\
180 \\
180\end{array}$ \\
\hline $\begin{array}{l}\text { ttt } \\
\text { tct } \\
\text { ctt } \\
\text { ctc } \\
\text { cct } \\
\text { ccc }\end{array}$ & $\begin{array}{l}1.354 \\
1.353 \\
1.354,1.354 \\
1.353 \\
1.354,1.350 \\
1.351\end{array}$ & $\begin{array}{l}\text { 6-31G ROH } \\
1.411 \\
1.414 \\
1.412,1.419 \\
1.420 \\
1.413,1.423 \\
1.425\end{array}$ & $\begin{array}{l}\text { HF } \\
1.398 \\
1.403 \\
1.398 \\
1.397 \\
1.404 \\
1.400\end{array}$ & $\begin{array}{r}180 \\
180 \\
0 \\
0 \\
24 \\
23\end{array}$ & $\begin{array}{r}180 \\
0 \\
180 \\
180 \\
16 \\
23\end{array}$ \\
\hline $\begin{array}{l}\mathrm{ttt} \\
\mathrm{tct} \\
\mathrm{ctt} \\
\mathrm{ctc} \\
\mathrm{cct} \\
\mathrm{ccc}\end{array}$ & $\begin{array}{l}1.375 \\
1.373 \\
1.375,1.374 \\
1.374 \\
1.376,1.371 \\
1.372\end{array}$ & $\begin{array}{l}6-31 G \text { CASS } \\
1.408 \\
1.410 \\
1.409,1.417 \\
1.417 \\
1.409,1.421 \\
1.422\end{array}$ & $\begin{array}{l}\text { SCF } \\
1.399 \\
1.404 \\
1.400 \\
1.399 \\
1.405 \\
1.402\end{array}$ & $\begin{array}{r}180 \\
180 \\
0 \\
0 \\
23 \\
22\end{array}$ & $\begin{array}{r}180 \\
0 \\
180 \\
180 \\
17 \\
23\end{array}$ \\
\hline
\end{tabular}

${ }^{a}$ For key to rotamer geometries, see Figure 1 . The bond lengths are converged to $\pm 0.0005 \AA$; two bond lengths that differ by this or less are set equal to the larger value upon rounding. ${ }^{b}$ Where the bonds on either end of the molecule are not symmetrically related, we report two values. 'For the STO-3G basis only planar geometries are examined. Since these are not minima for the cct and ccc structures we do not report them here.

rotamers had $C_{2 v}$ geometries; the remaining rotamers all had lower symmetry. All calculations were performed using GAMESs. ${ }^{13}$ Two different basis sets were used, the STO-3G basis ${ }^{14}$ and the 6-31G basis. ${ }^{15 a}$ In the study ${ }^{8 d}$ of butadiene ${ }^{+}$it was found that STO-3G excitation energies tended to be $0.2-0.3 \mathrm{eV}$ higher than the $6-31 \mathrm{G}$ results, and that augmentation of the 6-31G basis with diffuse or $\mathrm{d}$ functions had an approximately $0.1-\mathrm{eV}$ effect on the excitation energies. Given the limited correlation treatment employed here, these latter effects were not considered critical.

Initial geometry searches were performed using restricted open-shell Hartree-Fock wave functions (ROHF). In our study of butadiene ${ }^{+}$it was found that ROHF wave functions, when optimized in $C_{1}$ symmetry, produced nonsymmetrical equilibrium geometries. At higher levels of correlation the ground-state equilibrium geometry was found to be $C_{2 h}$; thus this artificial symmetry breaking was a result of the wave function attempting to correlate the three $\pi$ electrons. In hexatriene ${ }^{+}$we found no evidence for symmetry breaking at the SCF level; that is, the ttt rotamer was found to be $C_{2 h}$ to within the limits of the numerical convergence used even when the geometry search was begun from a $C_{1}$ configuration. Correlation was included using completeactive-space self-consistent field (CASSCF) wave functions where all $\pi$ electrons were distributed among the six lowest $\pi$ valence orbitals. In some cases the rotamers are distinctly nonplanar; nevertheless the three highest occupied orbitals still are essentially $\pi$-like, as are the three correlating orbitals obtained in the CASSCF treatment.

Excitation energies were based on CASSCF results for each state involved in the transition. The excitation energies reported correspond to vertical transitions from the equilibrium geometry for the ground electronic state of the given rotamer.

\section{Results}

In Table I the $\mathrm{C}-\mathrm{C}$ bond lengths and the central dihedral angles about the inner three $\mathrm{CC}$ bonds are presented at the calculated equilibrium geometries for each rotamer. In all cases the $\mathrm{C}-\mathrm{H}$ bonds were between 1.07 and $1.10 \AA$. Figures 1 and 2 show the 6-31G ROHF geometries. The numbering scheme for the carbon<smiles>[2H]OC(O)OC(O)OC(O)OC(O)O</smiles>

ttt<smiles>[2H]OC(O)OC(O)OC(O)OO</smiles>

$c t t$<smiles>[2H]OC(O)C(O)C(O)C(O)OC(O)O</smiles>

tct

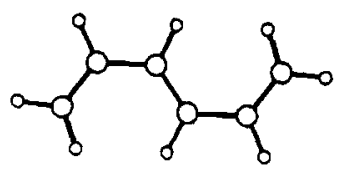

ctc
Figure 1. Geometries for the ttt, tct, ctt, and ctc rotamers of hexatriene ${ }^{+}$.<smiles>CC(O)C(C)OC(O)C(O)O</smiles><smiles>OC1C(O)C(O)C(O)C(O)C(O)C1O</smiles><smiles>OC1OC2OC3OC(O2)C(O1)C3O</smiles><smiles>OC1C(O)C(O)C(O)C(O)C(O)C1O</smiles>

$\operatorname{cct}$

$\mathrm{ccc}$

Figure 2. Geometries for the cct and cce rotamers of hexatriene ${ }^{+}$, from the side and from above.

atoms begins at one end and numbers consecutively across the molecule. Four of the rotamers, $\mathrm{ttt}$, tct, ctt, and ctc, are found to be planar, whereas the cce and cct rotamers are found to deviate significantly from planarity. This is in contrast to 6-31G RHF calculations on the neutral molecule by Yoshida et al. ${ }^{16}$ For the neutral molecule they found only two planar rotamers (ttt and tct) and found the energy ordering ttt, tct, ctt, cct, ctc, and ccc. We have performed optimizations for the cct and ccc rotamers where they are restricted to planarity and found that the energies of these stationary points at the 6-31G ROHF level are higher than the nonplanar results by 1.3 (cct) and $7.5 \mathrm{kcal} / \mathrm{mol}$ (ccc). Whether in the minimum basis set or the valence double- $\zeta$ set one finds quite similar results, and correlation of the $\pi$ electrons yields quite small changes in the computed geometries. In general, the CASSCF results lead to somewhat longer bond lengths than the ROHF results, in either basis.

To test the sensitivity of the above geometries to augmentation of the basis set with polarization functions, we have reoptimized the $\mathrm{ttt}$, ctc, and coc rotamers in the $6-31 \mathrm{G}^{* *}$ basis ${ }^{15 \mathrm{~b}}$ at the ROHF level (results not shown). We found that the end and central $C-C$ bonds decrease in length by approximately $0.005 \AA$ and that the two other inner bonds $\left(C_{2}-C_{3}\right.$ and $\left.C_{4}-C_{5}\right)$ increase by about the same amount. Small changes in the dihedral angles (about $2^{\circ}$ ) are also observed. In this larger basis the relative energy difference between ttt and ctc is $8.13 \mathrm{kcal} / \mathrm{mol}$ and the relative energy difference between ttt and $\mathrm{ccc}$ is $14.41 \mathrm{kcal} / \mathrm{mol}$. Little change in the relative energies is seen compared to the $6-31 \mathrm{G}$ results, and we have not pursued results in the larger basis any further.

We performed vibrational analyses for all rotamers at the 6-31G ROHF level, and each structure was shown to be a true minimum. In general the lowest vibrational frequency was on the order of $120 \mathrm{~cm}^{-1}$, except for ctc, where the lowest frequency was about $65 \mathrm{~cm}^{-1}$. The similarity of the geometrical parameters for the various levels of calculation employed here, coupled with the 
TABLE II: Relative Energies of Various Rotamers of Hexatriene ${ }^{+a}$

\begin{tabular}{lccccc}
\hline \multicolumn{1}{c}{ method } & tct & ctt & ctc & cct & ccc \\
\hline STO-3G ROHF $^{b}$ & 1.85 & 2.93 & 5.79 & & \\
STO-3G CASSCF $^{b}$ & 2.14 & 2.48 & 5.07 & & \\
6-31G ROHF & 2.27 & 3.80 & 7.57 & 8.53 & 14.18 \\
6-31G CASSCF & 2.70 & 3.37 & 6.90 & 8.62 & 13.99
\end{tabular}

${ }^{a}$ All energies are for the ground state of the molecule in the given configuration. Energies reported in $\mathrm{kcal} / \mathrm{mol}$ relative to the ttt rotamer in the given basis set and wave function. The energies of the ttt rotamers used are STO-3G ROHF, -228.76496 hartrees; STO-3G CASSCF, -228.85212 hartrees; 6-31G ROHF, -231.47014 hartrees; and 6.31G CASSCF, -231.53481 hartrees. ${ }^{b}$ Only planar configurations were considered in the STO-3G basis. Since these are not minima for the cct and ccc rotamers, the results are not reported here.

TABLE III: Excitation Energies for Various Rotamers of Hexatriene $^{+a}$

\begin{tabular}{ccccc}
\hline rotamer & $\Delta E_{1}(6-31 \mathrm{G})$ & $\Delta E_{2}(6-31 \mathrm{G})$ & $\Delta E_{1}(\mathrm{STO}-3 \mathrm{G})$ & $\Delta E_{2}(\mathrm{STO}-3 \mathrm{G})$ \\
\hline $\mathrm{ccc}$ & 2.15 & 3.30 & & \\
$\mathrm{cct}$ & 2.14 & 3.34 & & \\
ctc & 2.06 & 3.49 & 2.22 & 3.54 \\
$\mathrm{tct}$ & 2.17 & 3.50 & 2.28 & 3.57 \\
$\mathrm{ctt}$ & 2.11 & 3.53 & 2.25 & 3.58 \\
$\mathrm{ttt}$ & 2.16 & 3.59 & 2.28 & 3.61
\end{tabular}

a The first two $\pi$ excitation energies (in $\mathrm{eV}$ ) from CASSCF calculations for each rotamer. The CASSCF optimized geometry for the ground state of the given rotamer was used in each case. STO-3G results are not reported for the cct and ccc rotamers, since they were only treated as planar species in this basis. The ground-state energies for each rotamer can be calculated from the data in Table II.

stability of the stationary points at the 6-31G ROHF level, suggests that these will be minima in larger basis sets and/or in more complete correlation treatments.

Table II contains relative energies for the various rotamers, in $\mathrm{kcal} / \mathrm{mol}$, for all stationary points and methods used in Table I. In each case the energies are reported relative to the ttt rotamer in the given basis and wave function. The ttt rotamer was found to be the most stable rotamer in all cases. Interestingly, the STO-3G basis tends to yield somewhat lower energy differences for the other rotamers relative to the $\mathrm{ttt}$ rotamer than the $6-31 \mathrm{G}$ results. In addition, inclusion of correlation tends to decrease the relative energy of the ctt and ctc rotamers, while raising that of the tct rotamer. The cct and cce rotamers yield less generalizable results. In any event one sees that, independent of basis set or correlation treatment, (1) all six rotamers are stationary points, (2) the relative ordering of the rotamers in the ground state is found to be ttt, tct, ctt, ctc, cct, and cce, and (3) two of the rotamers are clearly nonplanar.

In Table III we present CASSCF excitation energies to the two lowest doublet $\pi$ excited states of hexatriene ${ }^{+}$at each of the stationary points located in Table I. It is seen that the excitation energies to the first excited state are quite similar, ranging by at most $0.1 \mathrm{eV}$ in either basis set. The second excitation energy varies by about $0.3 \mathrm{eV}$. The STO-3G results are about $0.05-0.10 \mathrm{eV}$ higher than the 6-31G results in all cases. Focusing on the second excitation (the one that has the greater intensity experimentally), the 6-31G CASSCF results predict the ordering ccc $<$ cct $<$ ctc $<$ tct $<\mathrm{ctt}<\mathrm{ttt}$. The STO-3G CASSCF results yield the same ordering for the four planar rotamers considered in this basis. However, the STO-3G results indicate that the tct and ctt excitations are nearly isoenergetic, whereas the 6-31G results predict that the ctc and tct transition energies are nearly identical. In the following discussion when ordering several transitions or when quoting experimental excitation energies, we have used the experimental intensity maxima. These are believed to correspond to the vertical transition from the ground-state geometry.

\section{Discussion}

Before discussing our results, it is useful to briefly review the experimental results and the analysis used in previous treatments of this system. In the earliest work, that of Shida et al. ${ }^{9}$ four separate spectra (other than that of the parent cyclohexadiene cation $\mathrm{CHD}^{+}$) were obtained by difference spectra after illumination of the cation. It was observed that three of the ions were formed concurrently upon illumination of $\mathrm{CHD}^{+}$, and that all three could eventually be converted into a species with a spectrum in agreement with that of $t t t$-hexatriene ${ }^{+}$. Through use of INDO and $\mathrm{CNDO} / \mathrm{S}$ calculations they produced potential energy surfaces for ground and excited states, and concluded that only production of ccc, ctc, tet, and ttt ions was consistent with the experiments and calculations. This mandated a simultaneous two-bond rotation about two inner carbon bonds in their reaction scheme. Later work by Kelsall and Andrews ${ }^{10}$ supported the experimental finding of four distinct species, and while they agreed with the assignment of the matrix spectrum of the ttt ion, they found no convincing experimental evidence that would allow assignment of the other spectra to particular rotamers.

Bally et al." also examined this system, but noted that gas-phase work ${ }^{17}$ found essentially identical first emission bands for the ttt and tct ions. Thus they were motivated to examine the possibility of overlapping bands for these species more closely. Furthermore, they eschewed the idea of a two-bond rotation after the primary photoreaction, and instead proposed that only one-bond rotations were occurring after photoreaction by the thermally activated ccc ion. They suggested that when a given species reacts, it initially only forms products that differ from it by rotation about one bond. This being the case, they assigned spectra to the ccc, cct, ctt, tct, and $t t t$ rotamers, suggesting that either ctc absorbed at longer wavelengths than all other species and was immediately photolyzed upon excitation, or that it was inherently less stable than the other species and underwent a quick thermal reaction. In the case of bleaching experiments of the rotamers, it is less clear on which surface the rearrangement takes place.

In regard to the calculations presented here, a few notes of caution should be added. First, our results are uniformly high for the calculated excitation energies. We believe that this most likely arises from not correlating the $\sigma$ electrons, but it is possible that other factors, such as the limited basis sets used, may be contributing to the high excitation energies observed. Second, a valid ordering of excitation energies will be based on a systematic cancellation of errors. In any calculation we neglect $\sigma$ correlation as well as $\sigma \pi$ correlation. In calculating an excitation energy we take a difference between energies, thus including a differential $\sigma$ and $\sigma \pi$ correlation error in the excitation energy. To the extent that this differential correlation error is not the same for all rotamers, we will obtain inaccurate estimates of the difference between excitation energies for two rotamers, and may even end up with an incorrect ordering of the rotamer excitation energies. Third, some of our excitation energies are quite similar, and in general one must be cautious in drawing conclusions based on small energy differences in any ab initio calculations, much less calculations where the correlation recovery is small and the basis set is limited. (See below for a detailed comparison with experiment.) Fourth, our results are best compared to gas-phase results, whereas most of the experiments are done in Freon or argon matrices. While the matrix shifts are small ${ }^{11}$ for these molecules, it is possible they may not be constant for all rotamers, thus influencing the relative order of the transitions. Fifth, while the above comments were aimed at the excitation energies, they could also be directed at the energy differences between the various ground-state stationary points. Thus, in examining our results and comparing them to experiment, we do so in the spirit that they may provide a useful guide or stimulus for new experiments, but they are by no means definitive calculations on these systems. Regarding the geometrical energy differences on the ground-state surface, it should be noted that in several examinations of the secondary minima for butadiene, double- $\zeta$ RHF calculations yield results within about $0.5 \mathrm{kcal} / \mathrm{mol}$ of larger basis set correlated calculations (see results in ref $12 a-c$ ). Since most of our energy differences are larger than $0.5 \mathrm{kcal} / \mathrm{mol}$, we believe that the ordering of the rotamer energies is reasonably accurate. 
With these cautions in mind then, we feel our results suggest the following. All six of the possible rotamers that have been postulated to exist for hexatriene ${ }^{+}$are found to be stationary points on the ground-state surface, and the 6-31G vibrational analyses indicate they are all true minima. Four of these species are planar, while two, those with adjacent cis bonds, are distorted from planarity. We find the lowest energy rotamer to be the ttt, followed by tct. Experimentally these are the two most stable rotamers for the neutral molecule. The energy ordering of the various rotamers is easily rationalized by asserting that the lowest energy rotamers have the greatest number of trans bonds, and that locating two cis linkages as far apart as possible lowers the energy of the rotamer (compare ctc and cct).

Considering the excitation energies for the various rotamers, we find that the first absorption bands are grouped closer together than the second absorption bands, in agreement with the experimental results. Our spreads in the range of excitation energies for both the first and second transitions are smaller than that observed experimentally; in fact, the first absorption bands all fall within $0.06 \mathrm{eV}$ in our results. This is clearly too small an energy difference on which to base assignments, and the ordering of the transitions will surely be susceptible to minor errors made in the differential correlation or basis sets used. The spread in the second absorption energies is $0.29 \mathrm{eV}$, and while still small, it is more likely that one might be able to make assignments based on these values.

In general terms, we find that the second transition energies increase as the number of trans linkages increases, with the ccc rotamer having the lowest transition energy and tt having the highest. This is in agreement with past deductions from experimental data, ${ }^{9-11}$ and this agreement lends support to the other excitation energies we report. (See below for comments on the geometry sensitivity of the present results and the estimated "error bars" for our energy differences.) We find the other four rotamers to have second transition energies between $\mathrm{ccc}$ and $\mathrm{ttt}$, and there seems no reason to believe that any one of them should be in greater error than the others. Thus, we would suggest that if any of these species was not observed in previous experimental studies, it was not due to an abnormally high or low transition energy. On the basis of the similarity of the transitions in our calculations, it may be possible that two or more species have such similar spectra that they overlap and appear as a single transition experimentally. The rotamer ctc was suggested to be the "missing" rotamer in the study by Bally et al. ${ }^{11}$ While its transition energy is not anomalous, it was found to have a significantly smaller torsional frequency than the other rotamers, and at higher levels of calculation it is possible that it is not a true minimum.

Somewhat more detailed experimental data are available for the $t t t$ and $t c t$ rotamers. In gas-phase photoionization experiments ${ }^{18}$ it is found that the energy difference between the first two ion states is essentially the same in the two rotamers (ttt, 1.95 $\mathrm{eV} ;$ tct, $1.97 \mathrm{eV}$ ). The photoionization results for the second $\pi$ excited state are obscured by overlapping bands. Emission spectra of the ions in the gas phase also find these transitions to be essentially isoenergetic (ttt, $1.968 \mathrm{eV}$; tct, $1.964 \mathrm{eV}$ ). ${ }^{17}$ Our results are in agreement with this finding. The matrix study of Bally et al. ${ }^{11}$ also finds nearly identical transition energies for the second band of these two rotamers, whereas we find a $0.1-\mathrm{eV}$ difference between the second transition energies for these two rotamers. To compare with the photoionization data, we have repeated CASSCF calculations on the three lowest ion states at the geometries of the neutral $\mathrm{ttt}$ and tct molecules. The geometries were obtained from similar CASSCF calculations in the 6-31G basis on the neutral species. At these geometries we find that for ttt the energy differences between ion states corresponding to the first two excitation energies of the ion are 2.13 and $3.45 \mathrm{eV}$, whereas for the tct rotamer the energy differences are 2.11 and $3.38 \mathrm{eV}$. The lowest IPs were calculated to be ttt, 7.81 ; and tct, $7.85 \mathrm{eV}$. (The experimental vertical IPs are ttt, 8.32; and tct, $8.29 \mathrm{eV}$ ). ${ }^{18}$ It is seen that at the neutral molecule geometries the first energy differences are nearly identical whereas the second differ by about $0.07 \mathrm{eV}$. We note however, that these energy differences, which
TABLE IV: Energy Ordering of the Second Electronic Transition for Various Rotamers

\begin{tabular}{ll}
\hline source & \multicolumn{1}{c}{ ordering } \\
\hline present results & $\mathrm{ccc}<\mathrm{cct}<\mathrm{ctc} \approx \mathrm{tct}<\mathrm{ctt}<\mathrm{ttt}$ \\
Shida et al. ${ }^{10}$ & $\mathrm{ccc}<\mathrm{ctc}<\mathrm{tct}<\mathrm{ttt}$ \\
Bally et al. ${ }^{12}$ & $\mathrm{ccc}<\mathrm{cct}<\mathrm{ctt}<\mathrm{tct} \approx \mathrm{ttt}$
\end{tabular}

${ }^{a}$ Ordering based on second transition energies from the $6-31 \mathrm{G}$ CASSCF calculations.

correspond to differences in ionization peaks, differ from our excitation energies at the ion geometries (corresponding to the vertical absorption peaks) by about $0.1 \mathrm{eV}$. Experimentally the photoionization energy differences, photoemission energies, and absorption energies ${ }^{19}$ (measured by photodissociation) are all found to be the same to within $0.02 \mathrm{eV},{ }^{17}$ In addition, we have reversed the ordering of the $\mathrm{ttt}$ and tct first excitation energies and IPs.

Thus our results appear to be somewhat too sensitive to geometry. However, we do find nearly identical first transition energies at these geometries, whereas the second transitions for $\mathrm{ttt}$ and tct are somewhat different, as was found at the ion equilibrium geometries. This suggests that our differing second excitation energies for the ttt and tct rotamers are not artifacts of the geometries used, and that modest errors in the ground-state geometries will not have a large effect on the ordering of the excitation energies in our results, except perhaps for transitions that are very close in energy (e.g., $\pm 0.02 \mathrm{eV})$. Taken together these results also indicate that our actual accuracy on energy differences between rotamers for a given transition is at best approximately $0.05-0.1 \mathrm{eV}$ (cf. the IP ordering). That is, in our calculations, energy differences of this order for a given transition may need to be regarded as a zero energy difference. However, the results may be better than the worst case that this error estimate would indicate, given that we do find tt to have the highest excitation energy, which is in agreement with experiment, and we find ccc to have the lowest transition energy, also in agreement with what has been deduced from experiment.

If one neglects the estimated error bars on energy differences for a given transition and takes the results at face value, one would suggest that the second transition energies are ordered as shown in Table IV, where we compare our predicted 6-31G CASSCF second excitation energy ordering to those of the experimental studies. It is seen that we produce the same general trend, placing all four remaining rotamers between the ccc and ttt rotamers, but differ in the ordering of the ctt and tct rotamers from the conclusions of Bally et al." In addition, while we obtained the same ordering as Shida et al., ${ }^{9}$ we find the ctc and tct second transitions nearly identical; thus it is quite difficult to be assertive about assignment of these two species based on our results.

One might hope that we could use the above results to assign all of the experimental transitions. While this appears possible for ttt and ccc, choosing which of the other rotamers have been observed does not seem feasible based on the current calculations. Although they do point to the probable existence of all six rotamers, they give little hint as to why they were all not observed in previous experiments, with the exception of our finding of a low torsional frequency for the ctc rotamer. One possible approach to this question may lie in examining the behavior of the three lowest $\pi$ states (and any intervening $\sigma$ states) as a function of rotation about various bonds. Another possibility is to attempt to calculate the transition moments for the first two transitions for the various rotamers.

Comparing the various theoretical methods used here, we find that the STO-3G and 6-31G basis sets yield essentially the same energetic ordering of the rotamers on the ground-state surface. This is a useful result for the treatment of larger polyene radical cations. For the calculation of excitation energies one also sees that the STO-3G CASSCF results are in good agreement with the 6-31G CASSCF results, again suggesting that one might obtain useful results in the smaller basis for larger molecules.

As mentioned above, other theoretical treatments of these rotamers have been performed. Shida et al. ${ }^{9}$ performed CNDO/S calculations for the low-lying states of ttt, tct, and ctc and found 
a larger spread in excitation energies for the first transition energy than for the second band. In addition, they predicted the ttt and ctc second transitions to be essentially degenerate, and found the tct second transition at higher energies than either ttt or ctc. This appears to be in disagreement with any of the experimental results, unless tct was not observed. Shida et $a .^{9}$ also used a PariserParr-Pople scheme for calculating excitation energies and with this method found a larger spread for the second transition than for the first, but significantly overestimated the individual excitation energies. In the PPP calculations they obtained the ordering $\mathrm{ctc}<\mathrm{tct}<\mathrm{ttt}$. Finally, SPINDO ${ }^{18}$ calculations have been used to interpret the photoionization spectra of trans- and cis-hexatriene. In addition to helping sort $\sigma$ and $\pi$ states, the method gave reasonably accurate ionization energies. ${ }^{18}$ The principle conclusion drawn from the study was the predicted similarity of the photoionization spectra for the two rotamers. Our results yield similar conclusions for the $\pi$ states examined here.

Finally, we have also performed geometry optimizations on the first two excited states of the ion for the four planar rotamer geometries at the CASSCF 6-31G level (results not shown). This allows one to theoretically estimate the difference between $0-0$ and vertical absorption peaks. For ttt, tct, ctc, and ctt we find planar stationary points for both the first and second excited states for each of the rotamers (vibrational analyses have not yet been performed to ascertain if these are true minima, but the optimizations were begun at slightly nonplanar geometries; yet the molecules remained essentially planar). The relaxation energy from the relevant ground-state geometry is $4.5 \pm 0.2 \mathrm{kcal} / \mathrm{mol}$ independent of state or rotamer. This translates to a difference between $0-0$ and vertical absorptions of about $0.2 \mathrm{eV}$ and emphasizes the similarity between $0-0$ and vertical transition energies. Given the overestimate of vibrational frequencies at the SCF level, one expects this to be an upper limit for the energy difference between the two transitions.

Conclusions. Results for ab initio calculations on various rotamers of hexatriene ${ }^{+}$have been presented. Six stationary points were found for the ground state of the cation, and vibrational analyses at the 6-31G ROHF level indicate these are true minima. Excitation energies based on energies obtained at the CASSCF level indicate the similarity between the transition energies in these rotamers. The calculations support the experimental assignments of the ttt and ccc rotamers and point up the similarity of the remaining rotamers. Our results indicate that assignment of the remaining species will be a difficult problem, both experimentally and theoretically.

Acknowledgment. This research was supported by a grant from the National Science Foundation, Grant No. CHE-9011770. J.L.J. was supported by a National Science Foundation REU grant (Grant No. CHEM-9100288) during the summer of 1991. The calculations were performed on the Harvey Mudd Computational Chemistry Facility, the establishment of which was made possible by a grant from the National Science Foundation, Grant No. USE-8950661.

Registry No. 1,3,5-Hexatriene radical cation, 60183-12-2.

\section{References and Notes}

(1) Birge, R. R. Annu. Rev. Biophys. Bioeng. 1981, 10, 315

(2) Bredas, J. L.; Street, G. B. Acc. Chem. Res. 1985, 18, 309.

(3) (a) Hudson, B. S.; Kohler, B. E.; Schulten, K. Excited States 1982 6, 1. (b) Hudson, B. S.; Kohler, B. E. Chem. Phys, Lett. 1972, 14, 299. (c) Hudson, B. S.; Kohler, B. E. J. Chem. Phys. 1973, 59, 4984. (d) Gavin, R M., Jr.; Weisman, C.; McVey, J, K.; Rice, S. A. J. Chem. Phys. 1978, 68, 522. (e) Hudson, B. S.; Kohler, B. E. Annu. Rev. Phys. Chem. 1974, 25, 437. (f) Buma, W. J.; Kohler, B. E.; Song, K. J. Chem. Phys. 1990, 92, 4622. (g) Ibid. 1991, 94, 6367. (h) Chadwick, R. R.; Gerrity, D. P.; Hudson, B. S Chem. Phys. Lett. 1985, 115, 24. (i) Mosher, O. A.; Flicker, W. M.; Kuppermann, A. J. Chem. Phys. 1973, 59, 6502. (j) Doering, J. P.; McDiarmid R. J. Chem. Phys. 1981, 75, 2477. (k) Granville, M. F.; Holtom, G. R. Kohler, B. E. J. Chem. Phys, 1980, 72, 4671. (1) Shang, Q.; Hudson, B. S Chem. Phys. Lett. 1991, 183,63.

(4) (a) Schulten, K.; Ohmine, I.; Karplus, M. J. Chem. Phys. 1976, 64, 4422. (b) Tavan, P.; Schulten, K. J. Chem. Phys. 1979, 70, 5407. (c) Lasaga A. C.; Aerni, R. J.; Karplus, M. J. Chem. Phys. 1980, 73, 5230.

(5) (a) Buenker, R. J.; Whitten, J. L. J. Chem. Phys. 1968, 49, 5381. (b) Hosteny, R. P.; Dunning, T. H., Jr.; Gilman, R. R.; Pipano, A.; Shavitt, I. J. Chem. Phys. 1975, 62, 4764. (c) Shih, S.; Buenker, R. J.; Peyerimhoff, S. D. Chem. Phys. Lett, 1972, 16, 244. (d) Buenker, R. J.; Shih, S.; Peyerimhoff, S. D. Chem. Phys. Lett. 1976, 44, 385. (e) Nascimento, M. A. C. Goddard, W. A., III. Chem. Phys. 1979, 36, 147. (f) Nascimento, M. A. C.; Goddard, W. A., III. Chem. Phys, 1980, 53, 251. (g) Nascimento, M. A. C. Goddard, W. A., III. Chem. Phys. Lett. 1979, 60, 197. (h) Cave, R. J.; Davidson, E. R.J. Phys. Chem. 1987, 91, 4481. (i) Cave, R. J.; Davidson, E. R. J. Phys. Chem. 1988, 92, 614. (j) Szalay, P. G.; Karpfen, A. Chem. Phys. 1989, 130, 219. (k) Cave, R. J.; Davidson, E. R. Chem. Phys. Lett. 1988, 148, 190. (l) Cave, R. J.; Davidson, E. R. J. Phys. Chem. 1988, 92, 2173 .

(6) Graham, R. L.; Freed, K. J. Chem. Phys. 1992, 96, 1304.

(7) (a) Shida, T.; Haselbach, E.; Bally, T. Acc. Chem. Res. 1984, 17, 180 (b) Bally, T.; Nitsche, S.; Roth, K.; Haselbach, E. J. Am. Chem. Soc. 1984, 106,3927 , and references cited therein.

(8) (a) Bigelow, R. W. Int. J. Quantum Chem. 1986, 24, 35. (b) Cederbaum, L. S.; Domcke, W.; Schirmer, J.; von Niessen, W.; Diercksen, G. H. F.; Kramer, W. P. J. Chem. Phys. 1978, 69, 1591. (c) Kimura, K.; Katsumata, S.; Achiba, Y.; Yamazaki, T.; Iwata, S. Handbook of HeI Photoelectron Spectra of Fundemental Organic Molecules; Halstead Press: New York 1981. (d) Cave, R. J.; Perrott, M. G. J. Chem. Phys. 1992, 96, 3745. (c) Dunkin, I. R. Tetrahedron $1985,4 l, 145$.

(9) Shida, T.; Kato, T.; Nosaka, Y. J. Phys. Chem. 1977, 81, 1095

(10) Kelsall, B. J.; Andrews, L. J. Phys. Chem. 1984, 88, 2723.

(11) Bally, T.; Nitsche, S.; Roth, K.; Haselbach, E. J. Phys. Chem. 1985, $89,2528$.

(12) (a) Breulet, J.; Lee, T. J.; Schaefer, H. F., III. J. Am. Chem. Soc. 1984, 106, 6250. (b) Szalay, P. G.; Lischka, H.; Karpfen, A. J. Phys, Chem. 1989, 93, 6629. (c) Alberts, 1. L.; Schaefer, H. F., IIl. Chem. Phys. Lett. $1989,161,375$.

(13) GAMESS is a general-purpose electronic structure program. The original program was assembled by M. Dupuis, D. Spangler, and J. J. Wendoloski at the National Resource for Computations in Chemistry, Software Catalog, University of California, Berkeley, CA, 1980; Program QGO1. The current version is described in the Quantum Chemistry Program Exchange Newsletter: Schmidt, M. W.; Baldridge, K. K.; Boatz, J. A.; Jensen, J. H.; Koseki, S.; Gordon, M. S.; Nguyen, K. A.; Windus, T. L.; Elbert, S. T. QCPE $B$ Bll. $1990,10,52$. The figures were produced using the MOLPLT package associated with GAMESS 2657 .

(14) Hehre, W. J.; Stewart, R. F.; Pople, J. A. J. Chem. Phys. 1969, 51,

(15) (a) Hehre, W. J.; Ditchfield, R.; Pople, J. A. J. Chem. Phys. 1972, 56, 2257. (b) Hariharan, P. C.; Pople, J. A. Theor. Chim. Acta 1973, 28, 213. (16) Yoshida, H.; Furukawa, Y.; Tasumi, M. J. Mol. Struct. 1989, 194, 279.

(17) Allan, M.; Maier, J. P. Chem. Phys. Lett. 1976, 43, 94.

(18) Beez, M.; Bieri, G.; Bock, H.; Heilbronner, E. Helv. Chim. Acta 1973, $56,1028$.

(19) Dunbar, R. J. Am. Chem. Soc. 1976, 98, 4670 